\title{
LDPCC and OFDM based cooperative communication for wireless sensor networks
}

\author{
Volkan Ozduran, Member, IEEE
}

\begin{abstract}
This paper investigates the low-density parity-check codes and orthogonal frequency division multiplexing technique effects on the relay assisted sensor networks. The investigation utilizes Rician and Rayleigh fading environments for the performance analysis. The investigation also considers two different types of information exchange processes, which are direct and relay assisted cases. In the relay assisted case, the relay terminal operates in amplify-and-forward and decode-and-forward modes. Monte-Carlo based computer simulations reveal that $10^{-5}$ bit error rate value is reached at $16.50 \mathrm{~dB}$ for low density parity check coded orthogonal frequency division multiplexing over Rician fading environment in decode-and-forward relay protocol. Similarly, $10^{-5}$ bit error rate value is reached at $0.36 \mathbf{d B}$ for the low density parity check coded orthogonal frequency division multiplexing over Rician fading environment in amplify-andforward relay protocol and finally $10^{-5}$ bit error rate value is reached at $-5.30 \mathrm{~dB}$ for low density parity check coded orthogonal frequency division multiplexing over Rician fading environment without relay protocols.
\end{abstract}

Index Terms-Low-density parity check-codes, OFDM, Cooperative communications, Wireless sensor networks

\section{INTRODUCTION}

$\mathrm{W}^{\mathrm{l}}$ ITH THE helping hand of advances in the sensor technology, wireless sensor networks have got a lot of usage areas in recent years. These usage areas can be summarized as: military, meteorological, biomedical, security, space exploration, monitoring, environmental, and home applications [1]. Although commonly usage of sensors, they have got some disadvantages. One of the main disadvantages of the sensors is the battery problem. These tiny creatures have got limited battery capacity. This negative way of the sensors effects to make better communication in low signal-to-noise ratio (SNR) values. Many researchers are dealing with this negative effect. One of the main desires for the communication systems is to achieve better communication values at lower power levels. In a classical point to point communication method, signals may lose in channel because of the fading effects. To prevent losing signal, it must be sent with high SNR values at transmitter side. This causes more energy consumption to reach better communication values.

On the other hand, cooperative communications [2] have got advantages over classical point to point communications. The basic idea behind cooperative communications is terminals

VOLKAN OZDURAN is with Department of Electrical and Electronics Engineering, Istanbul University-Cerrahpasa, Istanbul, Turkey, (email: volkan@istanbul.edu.tr).

https://orcid.org/0000-0002-9442-9099

Manuscript received February 12, 2020; accepted June 08, 2020.

DOI: $10.17694 /$ bajece.688294 share their resources to mitigate the fading and interference effects over signals. There are several cooperative relay strategies proposed in literature such as, amplify-and-forward (AF) [3], decode-and-forward (DF) [4], compress-and-forward [5], coded cooperation [6], and filter-and-forward [7]. One of the main objectives of these cooperation techniques is to use the relay node effectively. At each relay node, signals regenerate from the relay process and this minimizes the effect of the channels over signals and provides reliable and robust communications in low power values. In [8], authors proposed a joint network-channel coding in cooperative communications model and one of the main aim of their proposed model is to achieve better bit error rate (BER) performance values. In this proposed model, both source and relay nodes have to send their data to the destination node. Similarly, in [9], the authors proposed a model to reduce the power consumption effectively. In this proposed model, each source node behaves as a single carrier transmitter and the other nodes, which are near the source node, work as relay nodes, which shift the signal from the source node to specific frequencies. Moreover, in [10] the authors designed a model for energy efficient rural applications in wireless sensor networks and they reached sufficient results for a cluster head to data gather node transmission through Nakagami- $n$ channel in wireless sensor network.

So as to minimize the energy consumption, [11] utilizes cooperative multiple-input multiple-output (MIMO) orthogonal frequency division multiplexing (OFDM) system model structure for wireless sensor networks. Likewise, [12] also deals with the energy minimization for sensor networks applications. Apart from the aforementioned studies, [12] takes into consideration the circuit energy consumption as well. In addition, [12] also shows that single input single output (SISO) systems may outperform the MIMO systems when the data rate and modulation scheme are fixed. In order to minimize the overall transmit power, [13] proposes and analyses joint power control and bit rate assignment for OFDM modulated two-way AF relay wireless sensor networks. [14] utilizes Low-Density Parity-Check (LDPC) error correcting codes for channel coding and investigates the various types of multicarrier modulation techniques, which are single-carrier frequency division multiple access (SC-FDMA) and orthogonal frequency division multiple access (OFDMA), effect on system total power consumption. [15] provides a systematic literature review regarding error correcting codes for wireless sensor networks. [15] seeks answers for the importance of the error correction codes for wireless sensor network applications in terms of energy, power, and performance. [16] utilizes quasicyclic LDPC code for improving the complexity of the encoder in wireless sensor node. 
Reference [17] considers wavelet based compress-andforward relay protocol and investigates the wavelet compression families Haar and Daubechies-4 techniques' performance evaluations in wireless sensor networks. In addition, [17] utilizes LDPC error correction codes at transmitter side and maximum-ratio combining (MRC) technique at receiver side. Results in [17] reveal that Haar achieves better performance than Daubechies-4. [18] provides performance comparison of the binary phase shift keying (BPSK) modulated error correction codes in additive white Gaussian noise (AWGN) environment. [18] shows that Reed-Solomon code achieves better BER performance than its counterparts. [19] proposes serially concatenated LDPC and turbo codes scheme for wireless sensor networks. [20] seeks an answer for the distance and frequency effects on the error correction codes' energy efficiency in wireless sensor networks.

Differently from aforementioned studies, in order to minimize the overall energy consumption, this paper utilizes LDPC error correcting codes for channel coding process. BPSK modulation and OFDM multicarrier modulation techniques are chosen to achieve better communication values at low SNR. The MRC technique is utilized at the receiver side to combine the received signals, which are coming from source and relay terminals. This proposed model is performed with and without OFDM and relay nodes over various channel types to measure the robustness and overall power consumption of the designed model.

The rest of the paper is organized as follows. In Section 2 , the LDPC codes, which are used for channel coding, are briefly described. In Section 3, the OFDM technique is briefly described. In Section 4, purposed cooperative communication bits sequene system model is described. In section 5 , simulation results are presented and finally in Section 6, performance results are briefly discussed.

\section{LOW DENSITY PARITY CHECK CODES}

LDPC codes are known as a linear block codes and was first introduced by Gallager [21] in 1962. But it has not been used for a long time until McKay and Neal [22] brought it to the light in 1992. After 1992, LDPC codes has found a lot of usage areas in literature and in daily life. LDPC codes have got two types. The first one is regular LDPC codes, which is introduced by Gallager in 1962, and the other one is irregular LDPC codes [23]. The differences between regular and irregular LDPC codes are the structure of the parity check matrix, H. In regular LDPC codes, the parity check matrix's columns and weights are produced constantly. On the other hand, in irregular LDPC codes, the parity check matrix's columns and weights are produced non-constantly. Irregular LDPC codes perform better than regular LDPC codes because of the distribution of 1 and 0 bits.

\section{ORTHOGONAL FREQUENCY DIVISION MULTIPLEXING}

Over the last decade, with the improvements on the Digital Signal Processers (DSP), OFDM has found a lot of usage areas in wideband communication over mobile radio FM channels, asymmetric digital subscriber lines (ADSL), high-speed digital subscriber lines (HDSL), very high-speed digital subscriber lines (VHDSL), digital audio broadcasting (DAB), digital video broadcasting (DVB) and HDTV terrestrial broadcasting [24]. In a single-carrier data transmission technique, the information bit sequences are sent on a single-carrier modulation system and if there is a burst occurring in a bit sequence it affects all the bit sequences. To overcome this negative effect Frequency Division Multiplexing (FDM) method is proposed. FDM divides the frequency bands into several sub channels and these sub channels are non-overlapping each other. This enables to use the frequency spectrum efficiently. In 1966 Chang [25] proposed orthogonality and overlapping methods for multicarrier modulation to use the frequency spectrum efficiently. OFDM is a special type of FDM technique and the concept of the OFDM is to divide the communication channel into several sub channels which is called in commonly subcarriers. These subcarriers are orthogonal and overlapping between each other. This orthogonality and overlapping enable to use spectrum very efficiently. Each subcarrier carries the one bit of the total information bits [26, 27]. The OFDM transmitter and receiver structures are depicted in figure 1 (a) and (b), respectively [24].

(a) OFDM Transmitter Block
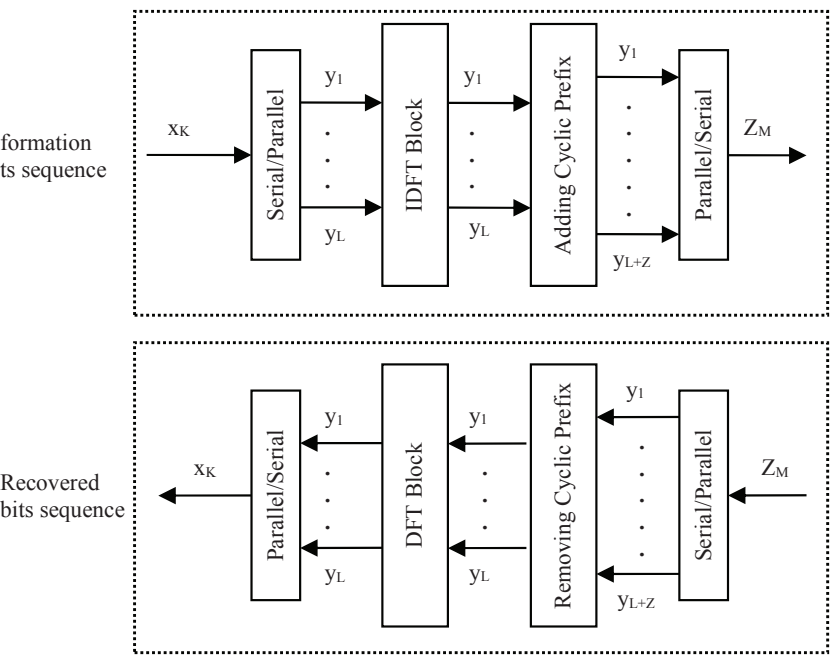

(b) OFDM Receiever Block

Fig. 1: Overview of OFDM scheme.

At transmitter side, a group of serial data sequences are made parallel by using serial to parallel converter and are sent to the IDFT block to modulate each separate parallel data sequences. Then these modulated bit sequences are sent to the cyclic prefix (CP) block to add bits from end of the line to avoid the inter-symbol interference (ISI) and inter-carrier interference (ICI) effects. After this process, these parallel bit sequences are sent to the parallel to serial blocks to be a serial data sequence. Finally, these data sequences are sent to the channel. At receiver side, serial data sequences are received 
and forwarded to the serial to parallel blocks and then sent to the removing $\mathrm{CP}$ block to remove added bits which are added at transmitter side to avoid the ISI and ICI. Then these bits are sent to the DFT blocks to demodulate and then sent to the parallel to serial block to handle serial data sequence.

\section{SYSTEM MODEL}

In proposed communication system models, which are depicted in figure 2 and 3 , the investigation considers with relay node and without relay node over various channel types such as, AWGN environment, fading environment both for Rician and Rayleigh types in wireless sensor networks. The investigation considers that the sensor nodes, which are presented in figure 2 and 3 , are placed in a small cluster with equal distance. The investigation utilizes LDPC codes for channel coding process instead of classical communication systems. This channel encoder helps to reach Shannon [28] theoretical communication limit at low signal to noise ratios. Since the relay terminal operates in half-duplex mode, the information exchange process can be completed in two phases, which are phase I and Phase II.

The received signals at relay and destination terminals for figure 2 and 3 can be written as:

$$
\begin{aligned}
& y_{s, r}=\sqrt{P_{1}} h_{s, r} x+n_{s, r} \\
& y_{s, d}=\sqrt{P_{1}} h_{s, d} x+n_{s, d}
\end{aligned}
$$

where $y_{s, d}$ and $y_{s, r}$ are received signals at destination and relay terminals, respectively. $\mathrm{P}_{1}$ is the transmit power at source terminal. $x$ is the transmit information. $h_{s, d}$ and $h_{s, r}$ are channel impulse responses between source $\rightarrow$ destination and source $\rightarrow$ relay, respectively. $n_{s, d}$ and $n_{s, r}$ are AWGN at destination and relay terminals, respectively.

In the case that relay terminal operates in AF mode, the relay terminal amplifies the received signal, which is coming from the source terminal, with $G$ amplification factor and forwards it to the destination terminal. The $\mathrm{G}$ amplification factor can be calculated as:

$$
G=\sqrt{\frac{\mathrm{P}_{2}}{\mathrm{P}_{1}\left|h_{s, r}\right|^{2}+N_{0}}}
$$

where $\mathrm{P}_{2}$ is the relay terminal's transmit power and $N_{0}$ is the noise variance at relay terminal. After this amplification process, the received signal at destination terminal can be written as:

$$
y_{r, d}=G \sqrt{\mathrm{P}_{2}} h_{r, d} y_{s, r}+n_{r, d}
$$

In the case that relay terminal operates in DF mode, the relay node receives coded signal coming from the source node and decodes the received signal and then transmits the decoded signal to the destination node. The received signal at destination terminal can be written as:

$$
y_{r, d}=\sqrt{\mathrm{P}_{2}} h_{r, d} \hat{x}+n_{r, d}
$$

where $\hat{x}$ is the decoded information. At destination node, the signals coming both from the source node and the relay nodes are combined by using MRC [29] or maximum-likelihood (ML) receiver [30]. The combined signal at the MRC detector can be written as in [29, 31]

$$
y=a_{1} y_{s, d}+a_{2} y_{r, d}
$$

Here, $y$ is the received signal both coming from the source node and the relay node. $a_{1}$ and $a_{2}$ factors can be formulated with the help of [29, 31] as:

$$
\begin{aligned}
& a_{1}=\frac{\sqrt{\mathrm{P}_{1}} h_{s, d}}{N_{0}} \\
& a_{2}=\frac{\sqrt{\mathrm{P}_{2}} h_{r, d}}{N_{0}}
\end{aligned}
$$

Cooperative communication system is designed based on following assumptions:

- (a) For LDPC structure, Generator matrix, $G$ is $302 x 1200$ bits and parity check matrix, $\mathrm{H}$ is $900 x 1200$ bits length and regular LDPC codes are used.

- (b) For OFDM structure, serial to parallel block divides the signal by 10 to make parallel and for the CP block takes 5 of the signal. For this model, 60 bits of the 1200 bits length of the signal for both adding CP and removing $\mathrm{CP}$ blocks.

- (c) For the channel type, when the communication system performed with the OFDM technique, noise is normalized with 0.08 coefficient.

- (d) For AF and DF cooperative relay protocols, transmit powers, $\mathrm{P}_{1}=0.6 P$ and $\mathrm{P}_{2}=0.4 P$ are chosen.

- (e) Both for AF and DF relay protocols 8 relay nodes are utilized for cooperative communication.

For no relay node model, communication system is designed by using LDPC codes with and without using OFDM technique and is performed over various environments such as AWGN environment, fading environment both for Rayleigh and Rician cases. This model is classical communication model and communication takes place between source node and destination node. At source node, 302 bit length source data is sent to the LDPC encoder block, after encoding process according to the assumption (a) at LDPC block, 302 bit length signal become 1200 bit length and sent to the BPSK modulation. The mathematical formulation for BPSK can be written as:

$$
z=2 x-1
$$

Here, $x$ is the 1200 bit length LDPC coded signal and $z$ is the output of the BPSK modulation. In BPSK modulation block "0" bits are converted to " -1 " and " 1 " bits are converted to the "1" and sent to the OFDM block. In OFDM block, modulated serial bits are converted to the parallel bits according to the assumption (b) at serial to parallel block. 1200 bits length signal is divided by "10" and we have $120 \times 10$ matrix and then this matrix block sent to the IDFT block. At IDFT block modulation process takes place here. After IDFT block, this signal set is sent to the CP block to add bits according to the assumption (b), which is stated above. The reason why these bits are added at CP block is to mitigate the effect of 


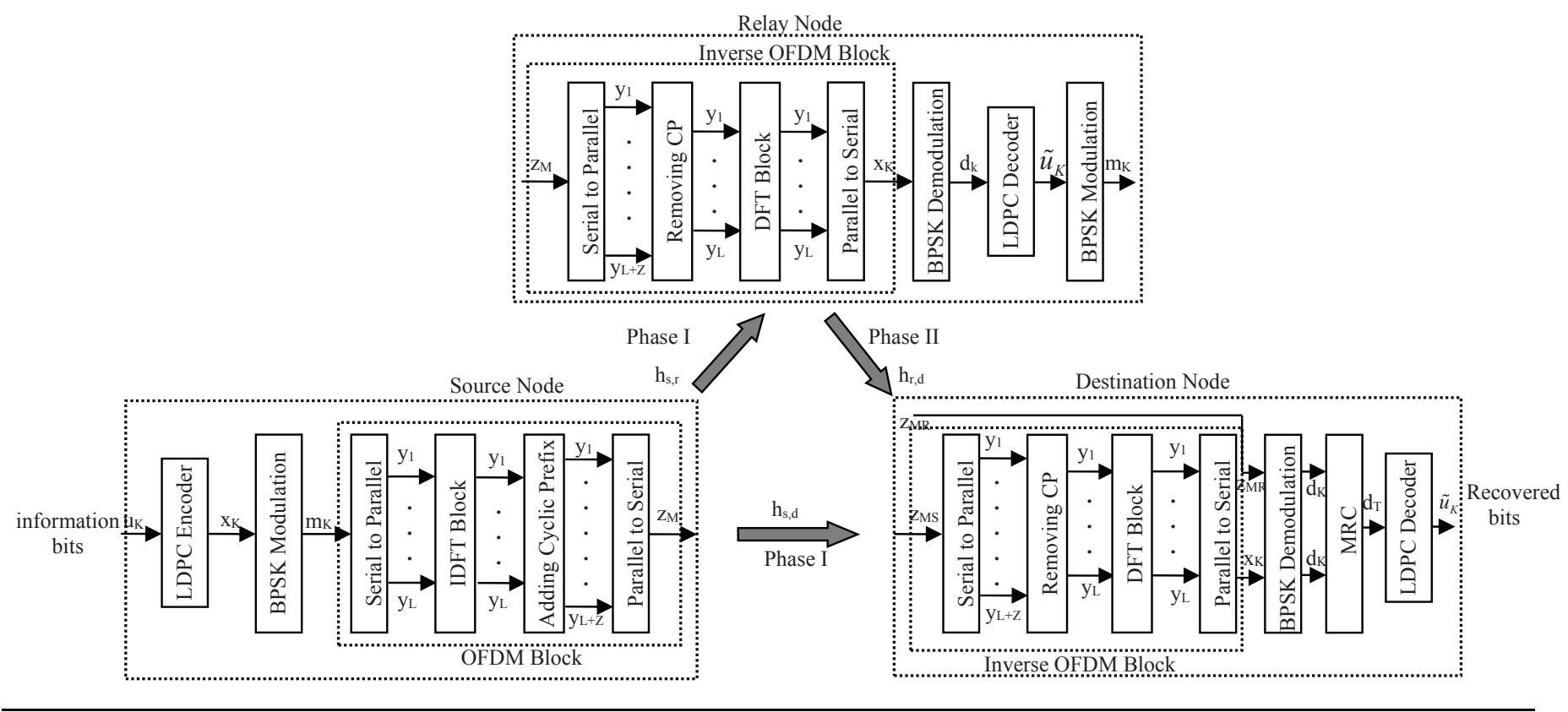

Fig. 2: DF based proposed cooperative communication model.

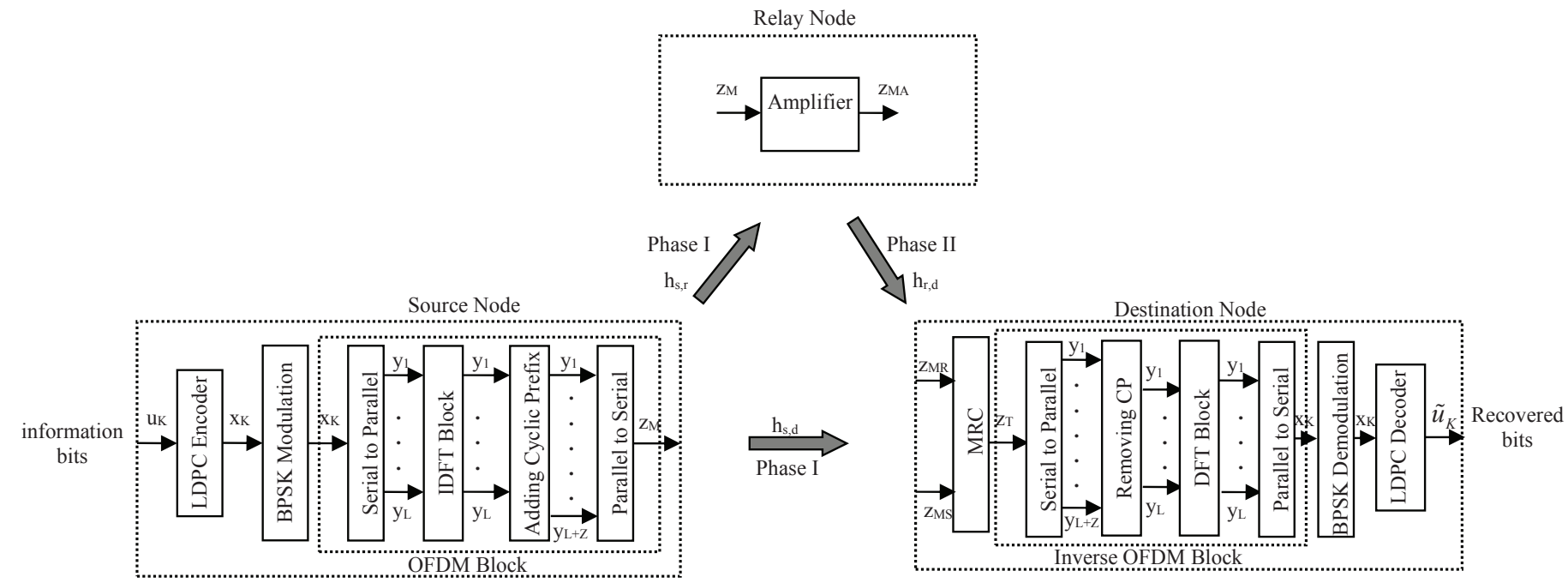

Fig. 3: AF based proposed cooperative communication model. 
the ISI and ICI. After adding CP block, signal set become a $126 x 10$ matrix and this parallel block is converted to serial bit set by parallel to serial block. This 1260 length bit set is sent to the channel. At channel the system is performed with AWGN environment and fading environment both for Rician and Rayleigh fading cases. When the communication system is performed with OFDM block, channel noise is normalized with 0.08 coefficient based on the assumption (c).

At receiver side, inverse OFDM block receives 1260 bit length noisy signal from channel. The first process in inverse OFDM block is to convert this 1260 bit length serial data stream into the parallel based on the assumption (b). After the serial to parallel block, signal set is become a $126 x 10$ matrix and is sent to the removing $\mathrm{CP}$ block to remove the $\mathrm{CP}$ bits, which is 60 bits. After the removing CP block signal set is become a $120 x 10$ matrix. This parallel block is converted to serial data stream by parallel to serial block and is become 1200 bit length. This block is the last block for the inverse OFDM block. 1200 bit length bit stream is demodulated with BPSK demodulation block and forward to the LDPC decoder block to handle the recovered bits. At LDPC decoder, Message Passing Algorithm (MPA) [32] is used for decoding process. After decoding process, recovered bit sequences are handled. For this proposed communication model, simulation results are presented in table 1 and figure 4.

For DF cooperative communication, system model is depicted in figure 2 and communication takes place in 2 phases, Phase I and Phase II. For phase I situation, source node is designed based on assumptions and likewise no relay model. Source node sends 1260 bit length bit stream to the relay node and destination node simultaneously according to (1) and (2), respectively. When the relay node receives noisy signal from source node, inverse OFDM block processes the signal and at the end of the inverse OFDM block, 1200 bit length set is handled and forwarded to the BPSK demodulation block. After demodulation process, LDPC decoder receives it and decodes it by using MPA and recovered bits are modulated with BPSK modulation block according to (9). For phase II situation, BPSK modulated signal is forwarded to the destination node according to (5).

Destination node receives these signals coming both from source node-phase I and Relay nodes-Phase II. These signals are different between each other, such as source node signal is LDPC coded OFDM signal and the relay node signal is decoded signal, so destination node process these signals separately. When the destination node receives noisy source signal from phase I case, it forwards to the inverse OFDM block and when the destination node receives decoded signal from relay nodes it forwards to the BPSK modulation block. These signal sets are demodulated separately and combined at MRC according to (6) and forwarded to the LDPC decoder. LDPC decoder decodes them using MPA and recovered bits are handled. Simulation results are presented in table 1 and figure 5 .

For AF cooperative communication, system model is illustrated in figure 3 and it is designed based on assumptions. Communication takes place in 2 phases, Phase I and Phase II as in DF protocols. Source node is similar with no relay model and DF model and for Phase I case, source node sends 1260 bit length bit stream to the relay node and destination node simultaneously according to (1) and (2), respectively. When the relay nodes receive noisy signal from source node they simply amplifies the noisy signal with (3) and forwards to the destination node according to (4) in Phase II case. Destination node receives signals both from source node and relay nodes and it combines these signals by using MRC according to (6) which is constructed based on (7) and (8). MRC block forwards to the inverse OFDM block and after the inverse OFDM blocks, 1260 bits length signal is become 1200 bits length and is forwarded to the BPSK demodulation block. Demodulated signal is sent to the LDPC decoder and it is decoded by using MPA and finally recovered bit sequences are handled. Simulation results are presented in table 1 and figure 6 .

\section{SIMULATION RESULTS AND DISCUSSION}

In this study, LDPC codes and OFDM techniques are utilized for cooperative wireless sensor networks. AF and DF relay protocols are chosen for cooperative communication model. The investigation is also modeled and performed LDPC coded OFDM and without OFDM technique with AWGN, fading environment both for Rician and Rayleigh cases without using any relay node. For no relay node case, the Shannon theoretical limit value, $10^{-5} \mathrm{BER}$, is reached at $-0.50 \mathrm{~dB}$ for LDPC coded AWGN environment, $-3.20 \mathrm{~dB}$ for LDPC coded OFDM over AWGN environment. The effect of the OFDM systems over performance evaluation can be clearly seen from performance curves. $2.70 \mathrm{~dB}$ gain is achieved by using OFDM system. Similarly when the LDPC coded signal is performed over fading environment, $10^{-5} \mathrm{BER}$ value is reached at $2.30 \mathrm{~dB}$ for Rayleigh fading case $(\mathrm{K}=0),-4.30 \mathrm{~dB}$ for LDPC coded OFDM over Rayleigh fading case. Here, 6.60 $\mathrm{dB}$ gain is reached by using OFDM system. For Rician fading case $(\mathrm{K}=10), 10^{-5} \mathrm{BER}$ value is reached with LDPC coded signal at $-0.10 \mathrm{~dB}$ and $-5.30 \mathrm{~dB}$ for LDPC coded OFDM system. Here, $6.40 \mathrm{~dB}$ gain is reached by using OFDM system. These performance evaluation curves are presented in figure 4. For DF, communication model is presented in figure 2 . The investigation is performed with and without OFDM systems. Here, $10^{-5}$ BER value is reached at $33.00 \mathrm{~dB}$ for LDPC coded Rayleigh fading environment case $(\mathrm{K}=0)$ and $30.00 \mathrm{~dB}$ for LDPC coded OFDM over Rayleigh fading environment. $3.00 \mathrm{~dB}$ gain is obtained by using OFDM. For Rician case $(\mathrm{K}=10), 10^{-5}$ BER value is reached at $19.00 \mathrm{~dB}$ for LDPC coded version and $10^{-5} \mathrm{BER}$ value is reached at $16.50 \mathrm{~dB}$ for LDPC coded OFDM over Rician fading environment. By using OFDM, $2.50 \mathrm{~dB}$ gain is obtained. Performance evaluation curves are presented in figure 5. For AF relay protocol, cooperative communication model is depicted in figure 3 . The investigation is performed with and without OFDM techniques. Performance curves are shown in figure 6 . Here, $10^{-5}$ BER value is reached at $1.42 \mathrm{~dB}$ for LDPC coded Rayleigh fading environment case $(\mathrm{K}=0)$. On the other hand, $0.75 \mathrm{~dB}$ is reached for LDPC coded OFDM over Rayleigh fading environment. In these cooperative communication schemes, by using OFDM, 


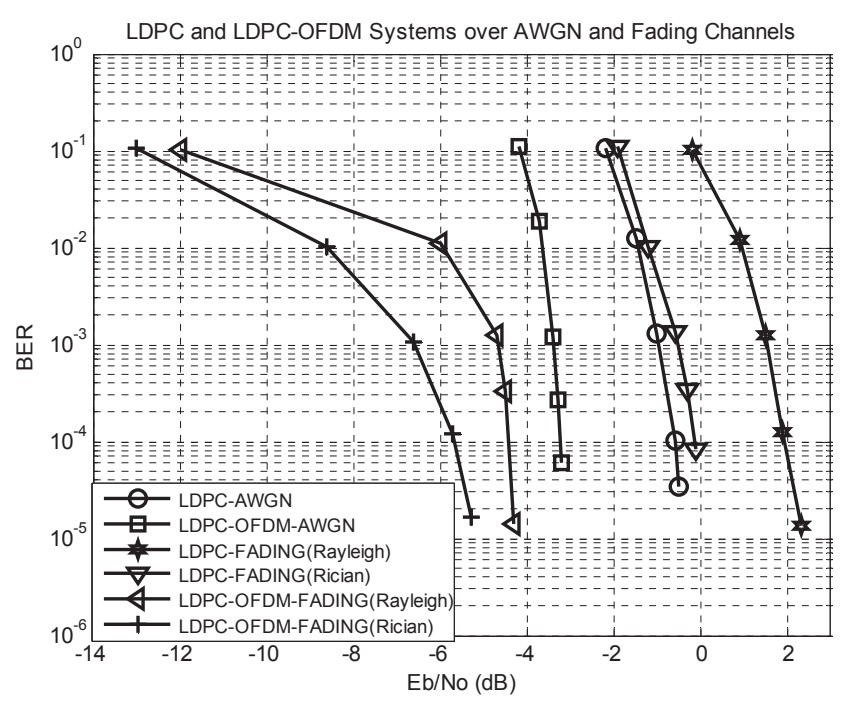

Fig. 4: Performance of LDPC and LDPC-OFDM Systems over AWGN and Fading Channels.

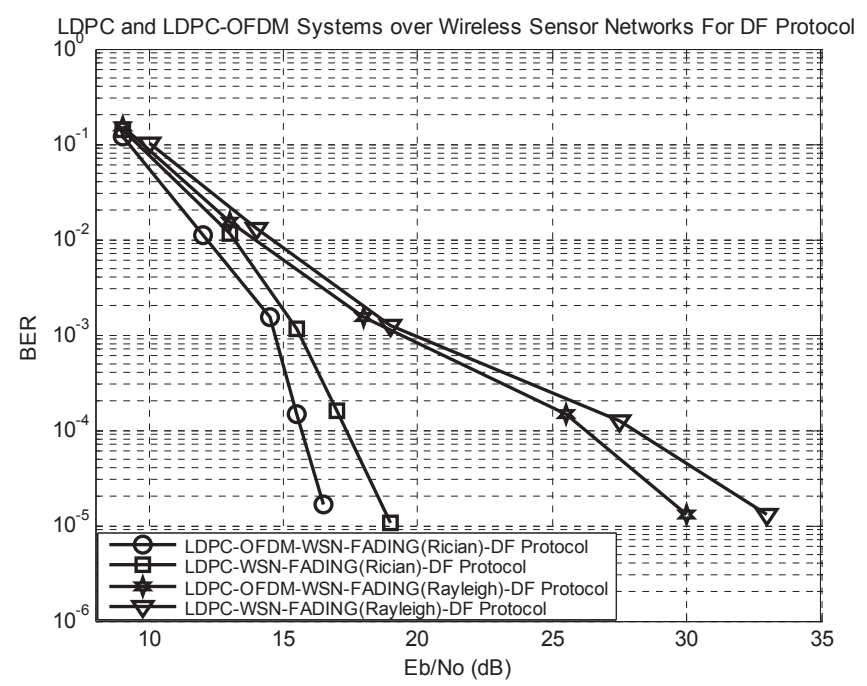

Fig. 5: Performance of LDPC and LDPC-OFDM systems over wireless sensor networks for DF relay protocols.

$0.67 \mathrm{~dB}$ gain is obtained. For Rician fading case $(\mathrm{K}=10), 10^{-5}$ BER value is reached at $1.09 \mathrm{~dB}$ for LDPC coded version and $10^{-5} \mathrm{BER}$ value is reached at $0.36 \mathrm{~dB}$ for LDPC coded OFDM over Rician fading environment. By using OFDM, $0.73 \mathrm{~dB}$ gain is obtained. Performance evaluation curves are presented in figure 6 .

According to the above simulation results, it can be clearly seen the effect of the OFDM systems over communication models. It is also observed from the figures that significant coding gain can be achieved by using OFDM and LDPCC techniques. When the relay protocols are performed in Rician

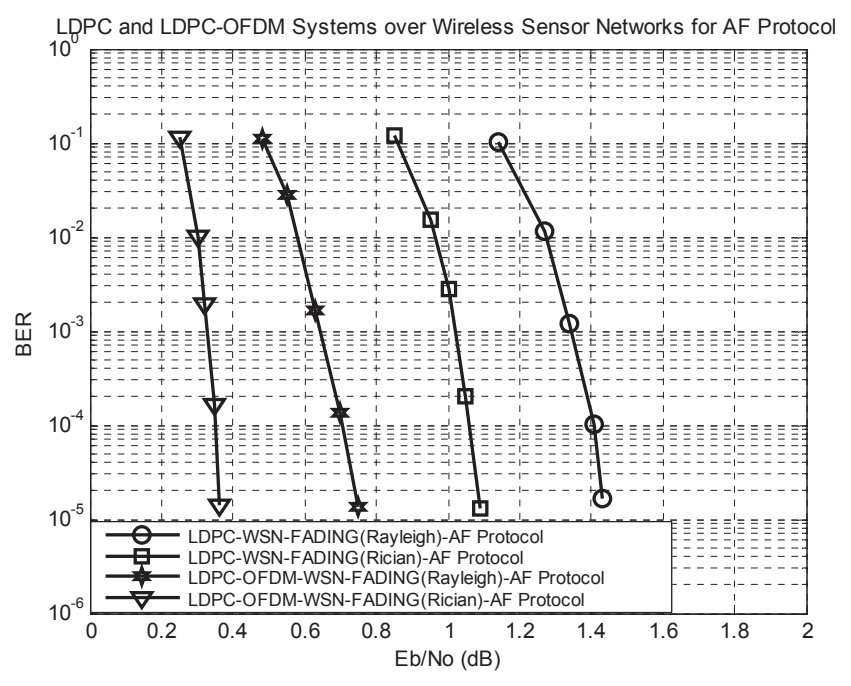

Fig. 6: Performance of LDPC and LDPC-OFDM systems over wireless sensor networks for AF relay protocols.

and Rayleigh fading cases, the best value is reached in AF relay protocol.

Consequently, for practical applications, AF relay protocol is better than DF relay protocol because of its lower complexity and lower power consumption.

\section{CONCLUSION}

This paper have proposed a communication model which utilizes LDPC encoder, OFDM, and communication protocols for the relay process, which are AF and DF protocols. The investigation have achieved significant coding gain by using OFDM technique. The results are clearly stated and observed the effect of the OFDM in the simulation results.

In future works, the proposed model can be compared by using different coding techniques, relay protocols and the multiplexing method, which is non-orthogonal multiple access, to achieve a better performance values in lower power consumption.

\section{REFERENCES}

[1] I. Akyildiz, W. Su, Y. Sankarasubramaniam, and E. Cayirci, "Wireless sensor networks: a survey," Computer Networks, vol. 38, no. 4, pp. 393 - 422, 2002.

[2] A. Nosratinia, T. E. Hunter, and A. Hedayat, "Cooperative communication in wireless networks," IEEE Communications Magazine, vol. 42, no. 10, pp. 74-80, Oct 2004.

[3] M. Torabi, W. Ajib, and D. Haccoun, "Performance analysis of amplify-and-forward cooperative networks with relay selection over rayleigh fading channels," in VTC Spring 2009 - IEEE 69th Vehicular Technology Conference, April 2009, pp. 1-5.

[4] J. N. Laneman and G. W. Wornell, "Distributed space-timecoded protocols for exploiting cooperative diversity in wireless networks," IEEE Transactions on Information Theory, vol. 49, no. 10, pp. 2415-2425, Oct 2003.

[5] S. Simoens, O. Munoz-Medina, J. Vidal, and A. Del Coso, "Compress-and-forward cooperative mimo relaying with full 


\begin{tabular}{|c|c|c|c|c|c|c|c|c|c|}
\cline { 2 - 10 } \multicolumn{1}{c|}{} & \multicolumn{3}{c|}{ AWGN Environment } & \multicolumn{7}{c|}{ Fading Environment } \\
\cline { 2 - 10 } \multicolumn{1}{c|}{} & LDPCC & LDPCC+OFDM & Gain & LDPCC & LDPCC+OFDM & Gain & LDPCC & LDPCC+OFDM & Gain \\
\hline No Relay & -0.50 & -3.20 & 2.70 & 2.30 & -4.30 & 6.60 & -0.10 & -5.30 & 6.40 \\
\hline AF Relay & - & - & - & 1.42 & 0.75 & 0.67 & 1.09 & 0.36 & 0.73 \\
\hline DF Relay & - & - & - & 33.00 & 30.00 & 3.00 & 19.00 & 16.50 & 2.50 \\
\hline
\end{tabular}

* This table is constructed based on the Shannon theoretical limits $10^{-5}$ Bit Error Rate (BER) value.

Fig. 7: The comparison results of without using any relay node, AF and DF protocols both for AWGN and Fading environments (in $\mathrm{dB}$ )

channel state information," IEEE Transactions on Signal Processing, vol. 58, no. 2, pp. 781-791, Feb 2010.

[6] T. E. Hunter and A. Nosratinia, "Performance analysis of coded cooperation diversity," in IEEE International Conference on Communications, 2003. ICC '03., vol. 4, May 2003, pp. 26882692 vol.4.

[7] H. Chen, A. B. Gershman, and S. Shahbazpanahi, "Filterand-forward distributed beamforming in relay networks with frequency selective fading," IEEE Transactions on Signal Processing, vol. 58, no. 3, pp. 1251-1262, March 2010.

[8] Du Bing and Zhang Jun, "Design and optimization of joint network-channel ldpc code for wireless cooperative communications," in 2008 11th IEEE Singapore International Conference on Communication Systems, Nov 2008, pp. 1625-1629.

[9] Weiguo Tang and Lei Wang, "Cooperative ofdm for energyefficient wireless sensor networks," in 2008 IEEE Workshop on Signal Processing Systems, Oct 2008, pp. 77-82.

[10] M. R. Islam and J. Kim, "Capacity and ber analysis for nakagami-n channel in ldpc coded wireless sensor network," in 2008 International Conference on Intelligent Sensors, Sensor Networks and Information Processing, Dec 2008, pp. 167-172.

[11] D. S. Singh A.K., Mishra S.K., "Energy efficiency in wireless sensor networks: Cooperative mimo-ofdm," Recent Trends in Communication, Computing, and Electronics. Lecture Notes in Electrical Engineering, vol. 524, no. -, pp. 147-154, December 2018.

[12] Shuguang Cui, A. J. Goldsmith, and A. Bahai, "Energyefficiency of mimo and cooperative mimo techniques in sensor networks," IEEE Journal on Selected Areas in Communications, vol. 22, no. 6, pp. 1089-1098, Aug 2004.

[13] X. Ji, Z. Bao, and C. Xu, "Power minimization for ofdm modulated two-way amplify-and-forward relay wireless sensor networks," J Wireless Com Network, vol. 70, no. -, pp. 1-8, April 2017.

[14] V. Ozduran and N. Ozdemir, "3gpp long term evolution (lte) based cooperative communication in wireless sensor networks," in 2012 IV International Congress on Ultra Modern Telecommunications and Control Systems, Oct 2012, pp. 900-905.

[15] M. Bettayeb, S. Ghunaim, N. Mohamed, and Q. Nasir, "Error correction codes in wireless sensor networks: A systematic literature review," in 2019 Intern. Conf. on Commun., Signal
Processing, and their Applications (ICCSPA), 2019, pp. 1-6.

[16] S. Choi and B. Moon, "Implementation of energy efficient ldpc code for wireless sensor node," Kim T. et al. (eds) Communication and Networking. FGCN 2011. Communications in Computer and Information Science, Springer, vol. 266, no. -, pp.,- 2011.

[17] V. Ozduran and O. N. Ucan, "Wavelet based compress-andforward relay protocol for cooperative communication in wireless sensor networks," in Computational Intelligence and Bioinformatics /755: Modelling, Simulation, and Identification, 2011.

[18] I. Ez-zazi, M. Arioua, A. el Oualkadi, and Y. el Assari, "Performance analysis of efficient coding schemes for wireless sensor networks," in 2015 Third International Workshop on RFID And Adaptive Wireless Sensor Networks (RAWSN), 2015, pp. 42-47.

[19] M. M. Salah and A. A. Elrahman, "Energy efficiency based concatenated ldpc and turbo codes for wireless sensor networks," in 2015 IEEE International Conference on Signal Processing, Communications and Computing (ICSPCC), 2015, pp. 1-6.

[20] S. Howard, C. Schlegel, and K. Iniewski, "Error control coding in low-power wireless sensor networks: When is ecc energyefficient?" J. Wireless Com Network, Springer, vol. -, no. -, pp. $1-14,2006$.

[21] R. Gallager, "Low-density parity-check codes," IRE Transactions on Information Theory, vol. 8, no. 1, pp. 21-28, January 1962.

[22] D. J. C. MacKay and R. M. Neal, "Near shannon limit performance of low density parity check codes," Electronics Letters, vol. 32, no. 18, pp. 1645-, Aug 1996.

[23] T. J. Richardson, M. A. Shokrollahi, and R. L. Urbanke, "Design of capacity-approaching irregular low-density parity-check codes," IEEE Transactions on Information Theory, vol. 47, no. 2, pp. 619-637, Feb 2001.

[24] A. Pandharipande, "Principles of ofdm," IEEE Potentials, vol. 21, no. 2, pp. 16-19, April 2002.

[25] R. W. Chang, "Synthesis of band-limited orthogonal signals for multichannel data transmission," The Bell System Technical Journal, vol. 45, no. 10, pp. 1775-1796, Dec 1966.

[26] L. Litwin and M. Pugel, "The principles of ofdm," RF signal processing, vol. -, no. 10, pp. 30-48, Jan 2001.

[27] H. Lotia, "Modeling a multicarrier wireless communication transceiver," Embedded Software systems literature survey, 
2004.

[28] C. E. Shannon, "A mathematical theory of communication," Bell System Technical Journal, vol. 27, no. 3, pp. 379-423, 1948.

[29] D. G. Brennan, "Linear diversity combining techniques," Proceedings of the IRE, vol. 47, no. 6, pp. 1075-1102, June 1959.

[30] X. Liu and W. Su, "Ber performance analysis of the optimum $\mathrm{ml}$ receiver for decode-and-forward cooperative protocol," in 2007 IEEE International Conference on Acoustics, Speech and Signal Processing - ICASSP '07, vol. 3, April 2007, pp. III-485-III488.

[31] W. Su, A. Sadek, and K. Ray Liu, "Cooperative communication protocols in wireless networks: Performance analysis and optimum power allocation," Wireless Pers Commun, vol. -, no. -, p. 181217, 2008.

[32] S. Arora, C. Daskalakis, and D. Steurer, "Message-passing algorithms and improved lp decoding," IEEE Transactions on Information Theory, vol. 58, no. 12, pp. 7260-7271, Dec 2012.

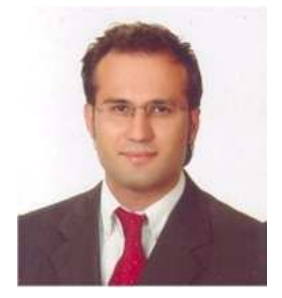

Volkan Ozduran graduated from department of Electronics at Soke Technical High School, Aydin, Turkey in 1997. He received his A.Sc. degree in Industrial Electronics, B.Sc., M.Sc. and Ph.D. degrees are in Electrical and Electronics Engineering from Istanbul University, Istanbul, Turkey in 2002, 2005, 2008 and 2015 respectively. During his Ph.D. studies he visited Stanford University, Stanford, CA, USA department of Electrical Engineering, Space, Telecommunications, and Radioscience Laboratory, Dynamic Spectrum Management (DSM) Research group as a visiting student researcher between April 2012 and October 2012 under the supervision of Prof. Dr. John M. CIOFFI, the best known father of DSL. Prof. CIOFFI was his formal second advisor in his $\mathrm{PhD}$ studies. During his Ph.D. studies he also had some short visits to California Institue of Technology (CALTECH), Pasadena, CA, USA and Princeton University, Princeton, NJ, USA, department of Electrical Engineering in April 2013 and November 2014, respectively. His current research interests area more on cooperative communications, interference mitigation, massive MIMO and signal processing for wireless communications. He is an active Reviewer in various IEEE conferences and Transactions Journals 